\title{
Autoantibodies against Matrix Metalloproteinase-1 in Patients with Localized Scleroderma
}

Saori Tomimura ${ }^{1}$, Fumihide Ogawa ${ }^{1}$, Yohei Iwata $^{1}$, Kazuhiro Komura ${ }^{1}$, Toshihide Hara ${ }^{1}$, Eiji Muroi $^{1}$, Motoi Takenaka ${ }^{1}$, Kazuhiro Shimizu ${ }^{1}$, Minoru Hasegawa ${ }^{2}$, Manabu Fujimoto ${ }^{2}$, and Shinichi Sato ${ }^{1}$

${ }^{1}$ Department of Dermatology, Nagasaki University Graduate School of Biomedical Sciences, Nagasaki, Japan and ${ }^{2}$ Department of Dermatology, Kanazawa University Graduate School of Medical Science, Kanazawa, Japan

Address correspondence to: Dr. Shinichi Sato, Department of Dermatology, Nagasaki University Graduate School of Biomedical Sciences, 1-7-1 Sakamoto, Nagasaki, 852-8501, Japan.

Phone: 81-95-819-7333

Fax: 81-95-849-7335

E-mail: s-sato@nagasaki-u.ac.jp

Short title: Anti-MMP-1 autoantibody in LSc 


\begin{abstract}
Background. Localized scleroderma (LSc) is characterized by cutaneous fibrosis and various autoantibodies.

Objective. To determine the presence or levels of antibodies (Abs) against matrix metalloproteinase (MMP)-1 and their clinical relevance in LSc.

Methods. Anti-MMP-1 Ab was examined by ELISA (Enzyme-Linked ImmunoSorbent Assay) and immunoblotting using human recombinant MMP-1. MMP-1 collagenase activity was determined using biotinylated collagen as substrate and the amount of cleaved biotinylated fragments of collagen by MMP-1 was measured by ELISA.

Results. LSc patients exhibited significantly elevated IgG anti-MMP-1 Ab levels relative to normal controls at similar level of patients with systemic sclerosis (SSc). However, IgG anti-MMP-1 Ab levels were comparable among the 3 LSc subgroups: morphea, linear scleroderma, and generalized morphea. When absorbance values higher than the mean + 2SD of normal controls were considered positive, IgG or IgM anti-MMP-1 Ab was found in 46\% and 49\% of total LSc patients and SSc patients, respectively. Anti-MMP-1 Ab was detected most frequently in morphea patients (60\%), followed by linear scleroderma patients (47\%) and then generalized morphea patients (25\%). LSc patients positive for IgG anti-MMP-1 Ab had elevated levels of IgG anti-single stranded DNA Ab, IgG anti-nucleosome Ab, and shorter disease duration relative to those negative. The presence of anti-MMP-1 Ab in LSc patients was confirmed by immunoblotting. IgG isolated from LSc patients’ sera positive for IgG anti-MMP-1 Ab by ELISA inhibited MMP-1 collagenase activity.
\end{abstract}

Conclusion. These results suggest that anti-MMP-1 autoantibody is a novel autoantibody in LSc. 


\section{INTRODUCTION}

Scleroderma, a chronic connective tissue disorder with an autoimmune background, is classified into localized scleroderma (LSc) and systemic sclerosis (SSc). LSc exhibits fibrosis limited to the skin and the subcutaneous tissues beneath the cutaneous lesions, and differs from SSc since LSc is not associated with Raynaud's phenomenon, acrosclerosis, and internal organ involvement [1]. Therefore, the prognosis for LSc patients is fairly good; however, the disfigurement and deformities of the extremities and face resulting from deep fibrosis significantly impair the quality of life [1]. Morphologically, LSc is classified into 3 subsets: morphea, linear scleroderma, and generalized morphea [2]. Morphea is usually characterized by one or a few circumscribed sclerotic plaques with an ivory-colored center and a surrounding violaceous halo. Linear scleroderma appears in a linear, band-like distribution, and often involves the muscle and bone underlying the skin lesions. Generalized morphea is a severe form of LSc characterized by widespread skin involvement with multiple lesions.

LSc exhibits autoimmunity as a central feature of the diseases, since antinuclear antibody (Ab) is detected in $46-80 \%$ of patients [3-5]. These autoantibodies react to various intracellular components, including nucleosome, histone, DNA topoisomerase II, and single-stranded DNA (ssDNA) [3,4,6-8] and also to some enzymes, such as superoxide dismutase (SOD) [9]. In addition, anti-cardiolipin Ab, rheumatoid factor, and lupus erythematosus cell phenomenon have been detected in LSc $[4,10]$. However, it remains unknown whether these autoantibodies directly contribute to the disease manifestations of LSc.

Both SSc and LSc show an abnormal accumulation of extracellular matrix (ECM) components, predominantly types I and III collagens [11-13]. The net accumulation of ECM in tissue is dependent on the balance between the synthesis and the degradation of ECM 
components. The latter process is regulated by matrix metalloproteinases (MMPs), a family of zinc-dependent endopeptidases that collectively can digest all ECM components [14,15]. MMP-1 (interstitial collagenase-1) is able to initiate degradation of interstitial collagen types I, II, and III [14]. A recent study has shown that autoantibody against MMP-1 is detected in $49 \%$ of patients with SSc, especially diffuse cutaneous type of SSc, but not in patients with other autoimmune disorders, such as systemic lupus erythematosus and dermatomyositis [16]. However, it remained unknown whether anti-MMP-1 Ab was also detected in LSc and whether it was related to some clinical manifestations. Therefore, we investigated the presence or levels of anti-MMP-1 Ab, its clinical correlation, and its functional significance in LSc patients. 


\section{MATERIALS AND METHODS}

\section{Serum samples}

Serum samples were obtained from 41 Japanese patients with LSc (11 males and 30 females). Patients were classified into the following 3 subgroups as described previously [2]: 12 patients with generalized morphea (2 males and 10 females), 19 patients with linear scleroderma (5 males and 14 females), and 10 patients with morphea ( 3 males and 7 females). The age of patients with LSc (mean \pm SD) was $25.7 \pm 17.6$ years old (generalized morphea, $22.8 \pm 19.8$; linear scleroderma, $18.6 \pm 10.1$; and morphea, $42.0 \pm 16.2$ years old). The disease duration of patients with LSc was $4.2 \pm 5.8$ years (generalized morphea, $3.4 \pm 2.8$; linear scleroderma, $4.8 \pm 7.1$; and morphea, $4.0 \pm 6.3$ years). None of LSc patients was treated with oral corticosteroid or other immunosuppressive therapy at the evaluation. The number of sclerotic lesions more than $3 \mathrm{~cm}$ in diameter was counted in each patient with LSc when the serum samples were obtained. The sclerotic lesions were morphologically classified into plaque and linear lesions. We divided the whole body into the following 7 areas: head and neck; right upper extremity; left upper extremity; anterior trunk; posterior trunk; right lower extremity; and left lower extremity, then we counted the number of involved areas as described previously [2]. Serum samples from 37 SSc patients who fulfilled the criteria proposed by the American College of Rheumatology [17] were also examined in this study. Thirty-four age- and sex-matched Japanese healthy persons were used as normal controls. Fresh venous blood samples were centrifuged shortly after clot formation. All samples were stored at $-70^{\circ} \mathrm{C}$ prior to use. The protocol was approved by the Kanazawa University Graduate School of Medical Science and Kanazawa University Hospital.

\section{Enzyme-Linked ImmunoSorbent Assay (ELISA) for anti-MMP-1 Ab}


ELISA was performed as previously described [16]. Briefly, 96-well plates were coated with human recombinant MMP-1 ( $1 \mu \mathrm{g} / \mathrm{ml}$; Techne Corp., Minneapolis, MN, USA) at $4^{\circ} \mathrm{C}$ overnight. Wells were blocked with $2 \%$ bovine serum albumin and $1 \%$ gelatin in Tris-buffered saline (TBS) for 1 hour at $37^{\circ} \mathrm{C}$. After washing twice with TBS, serum samples diluted to 1:100 in TBS containing $1 \%$ bovine serum albumin were added to triplicate wells and incubated for 90 minutes at $20^{\circ} \mathrm{C}$. After washing 4 times with TBS containing $0.05 \%$ Tween-20, plates were incubated with alkaline phosphatase-conjugated goat anti-human IgG or IgM Abs (Cappel, Durham, NC, USA) for 1 hour at $20^{\circ} \mathrm{C}$. After washing 4 times with TBS containing $0.05 \%$ Tween-20, substrate solution containing $0.91 \mu \mathrm{g} / \mu \mathrm{l}$ p-nitrophenyl phosphate (Sigma-Aldrich Co., St. Louis, MO, USA) in diethanolamine buffer ( $1 \mathrm{M}$ diethanolamine, $0.5 \mathrm{M} \mathrm{MgCl}_{2}$ ) was added and the optical density (OD) of the wells at $405 \mathrm{~nm}$ was subsequently determined. Absorbance values greater than the mean + 2SD of normal controls were considered positive in this study.

\section{Detection of antihistone Ab, anti-ssDNA Ab, anti-nucleosome Ab, and rheumatoid factor}

ELISA for antihistone and anti-ssDNA Ab was performed as described previously [4]. Briefly, 96-well microtiter plates were coated with total histones ( $5 \mu \mathrm{g} / \mathrm{ml}$; Sigma-Aldrich Co.) at $4^{\circ} \mathrm{C}$ overnight. For anti-ssDNA Ab, wells were pretreated with $0.1 \%$ protamine sulfate (grade X; Sigma-Aldrich Co.) for 1 hour at $20^{\circ} \mathrm{C}$. After rinsing, the plates were coated with calf thymus ssDNA ( $1 \mu \mathrm{g} / \mathrm{ml}$; Sigma-Aldrich Co.) at $4^{\circ} \mathrm{C}$ overnight. ELISA was performed as described for anti-MMP-1 Ab ELISA. Anti-nucleosome Ab was determined by commercial ELISA kits (D-TEK, Mons, Belgium), according to the manufacturer's protocol. Rheumatoid factor was measured using a latex agglutination slide test (Eiken, Tokyo, Japan), according to the manufacture's protocol. 


\section{Immunoblotting}

Human recombinant MMP-1 (0.1 $\mu \mathrm{g} / \mathrm{lane}$; Techne Corp.) was subjected to electrophoresis and electrotransferred to nitrocellulose sheets. The nitrocellulose strips were incubated overnight with serum samples diluted 1:50 and were then incubated for 1.5 hours with alkaline phosphatase-conjugated goat anti-human IgG Ab (Cappel). Color was developed using 5-bromo-4-chloro-3-indrolyl phosphate and nitro blue tetrazolium (Sigma-Aldrich Co.). Ten LSc patients positive for IgG anti-MMP-1 Ab by ELISA, 5 patients with LSc negative for IgG anti-MMP-1 Ab by ELISA, and 5 healthy individuals were evaluated.

\section{MMP-1 collagenase activity assay}

IgG was purified from serum samples using magnetic beads coated with recombinant Protein $G$ covalently coupled to the surface (Dynal Inc., Lake Success, NY, USA). Final IgG concentration was measured by spectrophotometer (Gene Quant II, Amarsham Biosciences Inc., Piscataway, NJ, USA). MMP-1 collagenase activity was determined by a collagenase activity kit (Chemicon International, Inc., Tremecula, CA, USA), according to the manufacture's protocol. Briefly, 4.5 ng of MMP-1 activated by $p$-aminophenylmercuric acetate was incubated with $40 \mu$ g of purified IgG for 20 minutes at $20^{\circ} \mathrm{C}$. The enzymatic activity of MMP-1 was measured using biotinylated bovine native collagen as substrate. The cleaved biotinylated fragments of collagen by MMP-1 were transferred to triplicate wells of biotin-binding 96-well microtiter plates and detected by streptavidin-peroxidase complex and enzyme substrate. The OD of the wells was subsequently determined. MMP-1 that was activated by p-aminophenylmercuric acetate and untreated with 
IgG served as positive control. Six LSc patients positive for IgG anti-MMP-1 Ab by ELISA and 6 healthy individuals were assessed.

\section{Statistical analysis}

Statistical analysis was performed using the Mann-Whitney $U$ test for determining the level of significance of differences between sample means, Fisher's exact probability test for comparison of frequencies, and Bonferroni's test for multiple comparisons. Spearman's rank correlation coefficient was used to examine the relationship between two continuous variables. A p value less than 0.05 was considered statistically significant. 


\section{RESULTS}

\section{Anti-MMP-1 autoantibody levels by ELISA}

The presence and levels of anti-MMP-1 autoantibodies in serum samples from patients with LSc or SSc and normal controls were assessed by ELISA (Figure 1). Patients with LSc exhibited significantly elevated IgG anti-MMP-1 Ab levels when compared with normal controls $(\mathrm{p}<0.001)$. Similarly, IgG anti-MMP-1 Ab levels in SSc patients were significantly higher than those found in normal controls $(\mathrm{p}<0.001)$. There was no significant difference in IgG anti-MMP-1 Ab levels between LSc and SSc patients. Regarding the disease subsets of LSc, IgG anti-MMP-1 Ab levels in patients with linear scleroderma and those with morphea were significantly higher than those found in normal controls ( $<<0.01$ and $\mathrm{p}<0.001$, respectively), while patients with generalized morphea did not exhibit significantly elevated IgG anti-MMP-1 Ab levels relative to normal controls. However, IgG anti-MMP-1 Ab levels were comparable among the 3 subgroups of LSc. By contrast, IgM anti-MMP-1 Ab levels in patients with total LSc, generalized morphea, linear scleroderma, morphea, or SSc were not significantly elevated compared to normal controls. Thus, IgG but not IgM anti-MMP-1 Ab levels were increased in both LSc and SSc at similar level.

Absorbance values higher than the mean + 2 SD (1.00 for IgG anti-MMP-1 Ab and 1.04 for IgM anti-MMP-1 Ab) of the control serum samples were considered positive in this study (Figure 1). In total patients with LSc, IgG or IgM anti-MMP-1 Ab was found in 46\% (Table 1). IgG isotype of this autoantibody (39\%) was more frequently detected than IgM isotype (10\%). Similar positivity was observed in SSc patients (49\%). In the subgroups of LSc, IgG or IgM anti-MMP-1 Ab was detected most frequently in patients with morphea (60\%), followed by patients with linear scleroderma (47\%) and then patients with generalized morphea (25\%). By 
contrast, IgG or IgM anti-MMP-1 Ab was not detected in any healthy individuals. Thus, IgG anti-MMP-1 Ab was detected in LSc and SSc at a similar frequency and the prevalence of IgG anti-MMP-1 Ab positivity was the highest in morphea.

\section{Clinical correlation}

Then, we assessed clinical correlation of anti-MMP-1 Ab in LSc patients. LSc patients positive for IgG anti-MMP-1 Ab had significantly elevated levels of IgG anti-ssDNA Ab $(\mathrm{p}<0.05)$ and IgG anti-nucleosome Ab ( $<<0.05$, Table 2). Consistent with this, IgG anti-MMP-1 Ab levels correlated positively with levels of IgG anti-ssDNA Ab $(\mathrm{r}=0.443, \mathrm{p}<0.01)$ and $\operatorname{IgG}$ anti-nucleosome $\mathrm{Ab}(\mathrm{r}=0.383, \mathrm{p}<0.05)$. Furthermore, the disease duration in LSc patients with IgG anti-MMP-1 Ab was significantly shorter relative to those without $(\mathrm{p}<0.05)$. However, IgG anti-MMP-1 Ab positivity did not correlate with any other clinical parameters. In addition, IgM anti-MMP-1 Ab positivity was not related to any clinical parameters (data not shown). Thus, the presence of IgG anti-MMP-1 Ab was associated with elevated levels of IgG anti-ssDNA Ab, IgG anti-nucleosome Ab, and shorter disease duration in LSc.

\section{Immunoblotting analysis for anti-MMP-1 Ab}

The presence of anti-MMP-1 Ab was further evaluated by immunoblotting analysis using human purified MMP-1. Serum samples from LSc patients positive for IgG anti-MMP-1 Ab by ELISA exhibited reactivity with MMP-1 ( 52 kDa) by immunoblotting (lanes 2-4, Figure 2). By contrast, serum samples from LSc patients negative for IgG anti-MMP-1 Ab by ELISA did not react with MMP-1 (lane 5). Furthermore, serum samples from healthy individuals did not react 
with MMP-1 (lane 6). Thus, the presence of anti-MMP-1 autoantibody in patients with LSc was confirmed by immunoblotting analysis.

\section{Inhibition of MMP-1 collagenase activity by IgG isolated from serum samples of LSc patients which contained IgG anti-MMP-1 Ab}

To determine the functional significance of anti-MMP-1 Ab, it was assessed whether anti-MMP-1 Ab was able to inhibit MMP-1 collagenase activity. MMP-1 collagenase activity was determined using biotinylated bovine native collagen as substrate. The amount of cleaved biotinylated fragments of collagen by MMP-1 was measured by ELISA. Six LSc patients positive for IgG anti-MMP-1 Ab by ELISA and 6 healthy individuals were evaluated. Relative OD values of IgG anti-MMP-1 Ab by ELISA in 6 LSc patients were 1.228, 1.260, 1.443, 1.468, 1.552, and 2.292. Furthermore, the presence of IgG MMP-1 Ab was confirmed by immunoblotting in all 6 LSc patients. MMP-1 collagenase activity was not inhibited by IgG isolated from healthy individuals (Figure 3). By contrast, IgG isolated from serum samples of LSc patients positive for IgG anti-MMP-1 Ab by ELISA significantly inhibited MMP-1 collagenase activity by $84.7 \%$ compared with normal control $(\mathrm{p}<0.05)$. There was no significant correlation between IgG anti-MMP-1 Ab levels by ELISA and the MMP-1 activity inhibition levels ( $\mathrm{r}=0.20$, $\mathrm{p}=0.68$ ). Thus, IgG anti-MMP-1 Ab from patients with LSc was able to inhibit MMP-1 collagenase activity. 


\section{DISCUSSION}

LSc is characterized by skin fibrosis with autoimmune background. Fibroblasts from the affected skin of LSc patients produce increased amount of collagen types I and III and fibronectin [13] [12] , which is also observed in fibroblasts derived from the SSc skin [11]. Furthermore, analysis using fibroblast cultures derived from different layers of the dermis and subcutaneous tissue has shown that the pattern of collagen synthesis in LSc is similar to that of SSc, suggesting that the two diseases have a common pathomechanism [18]. In SSc, a previous study has shown that SSc fibroblasts exhibit decrease in MMP-1 activity and production compared with normal fibroblasts [19]. Likewise, decrease in MMP-1 expression is observed in the LSc fibroblasts [20]. Moreover, the efficacy of ultraviolet A phototherapy on skin fibrosis is mainly obtained by increased production of MMP-1 and interferon- $\gamma$ in LSc [21]. Collectively, these findings suggest that MMP-1 expression and activity play a role in the development of skin fibrosis in both LSc and SSc. In the current study, anti-MMP-1 autoantibody was detected in LSc to a similar level and frequency of SSc. The presence of anti-MMP-1 Ab was further confirmed by immunoblotting analysis. The finding that anti-MMP-1 Ab levels correlated with levels of anti-ssDNA Ab and anti-nucleosome $\mathrm{Ab}$ suggests that anti-MMP-1 $\mathrm{Ab}$ is produced by autoimmune responses common to the production of other autoantibodies in LSc.

Both LSc and SSc exhibit autoimmunity as a central feature of the diseases. SSc exhibits the production of autoantibodies against various intracellular components, such as DNA topoisomerase I, centromere, RNA polymerases, and U3RNP [22]. However, these autoantibodies specific to SSc are absent in LSc with few exceptions [4]. Conversely, autoantibodies against DNA topoisomerase II and SOD, which are detected in LSc, are not found in SSc [8,9]. Thus, although both SSc and LSc show cutaneous fibrosis, autoantibody 
specificities associated with these diseases are different. However, LSc and SSc share some autoantibody specificities, such as antihistone $\mathrm{Ab}$, anti-ssDNA Ab, and rheumatoid factor. Similarly, anti-MMP-1 Ab is detected in both LSc and SSc. Furthermore, anti-MMP-1 Ab is not observed in other autoimmune diseases, including systemic lupus erythematosus and dermatomyositis, suggesting that anti-MMP-1 Ab is specific to LSc and SSc. Therefore, anti-MMP-1 Ab may be an autoantibody closely related to skin fibrosis.

A majority of autoantibodies that have been detected in LSc appear to correlate with the disease severity of LSc: antihistone $\mathrm{Ab}$, anti-ssDNA $\mathrm{Ab}$, and anti-cardiolipin $\mathrm{Ab}$ are most frequently positive in generalized morphea, the most severe subset of LSc, compared to linear scleroderma and morphea [4,10]. Especially, antihistone Ab correlates with total number of lesions and the total area of lesions [2]. On the other hand, autoantibodies against SOD or DNA topoisomerase II are found at comparable frequency between the 3 subgroups of LSc [8,9]. By contrast, the present study showed that anti-MMP-1 Ab was most frequently detected in morphea (60\%), followed by linear scleroderma (47\%) and then generalized morphea (25\%). The reasons for this phenomenon remained unknown in this study. However, the early diagnosis of morphea is sometime difficult, since early lesions of morphea occasionally show non-specific inflammatory change rather than sclerotic change. Therefore, the presence of anti-MMP-1 Ab would be helpful for diagnosing early stage of morphea. Moreover, LSc patients with anti-MMP-1 Ab exhibited shorter disease duration relative to those without, suggesting that anti-MMP-1 Ab is produced in the early phase of LSc. This finding would also help early detection of morphea.

In SSc, IgG anti-MMP-1 Ab correlates with diffuse cutaneous type of SSc and the extent of skin and lung fibrosis [16]. Furthermore, IgG anti-MMP-1 Ab in sera from SSc patients 
inhibits MMP-1 collagenase activity [16], suggesting that IgG anti-MMP-1 Ab contributes to the accumulation of ECM in SSc. Similarly, the current study showed that IgG anti-MMP-1 Ab in sera from LSc patients inhibited MMP-1 collagenase activity. However, the presence of IgG anti-MMP-1 Ab did not correlate with total number of lesions and the total area of lesions in LSc. Unlike SSc, LSc is characterized by the scattered sclerotic lesions that do not involve the whole body even if the lesions are multiple. Therefore, it remained unknown why systemic production of autoantibodies against MMP-1 in LSc affects only partial body areas, given that the autoantibodies can block collagenase activity of MMP-1. It has been reported that various physical injury, including trauma, radiation, burn, local corticosteroid injection, surgical procedures, immunization, and varicella infection, is a provoking factor in LSc [23,24]. In addition, MMP-1 expression is induced in response to exogenous signals, such as various cytokines or growth factors, cell-matrix interactions, and cell-cell interactions [14,15]. Indeed, a recent study has shown that MMP-1 expression is induced during the process of wound healing [25]. Therefore, it may be possible that physical skin injury induces the local expression of MMP-1, activity of which may be inhibited by anti-MMP-1 autoantibodies in LSc, suggesting that anti-MMP-1 autoantibodies may induce collagen deposition in LSc. However, since immunoglobulin deposition was not detected in the lesional dermis of LSc patients positive for IgG anti-MMP-1 Ab (data not shown), it remained unknown whether anti-MMP-1 Ab exhibited in vivo functional significance in LSc. Nonetheless, the results of this study indicate that anti-MMP-1 Ab is a novel autoantibody in LSc. 
Table 1. Frequency of anti-MMP-1 Ab positivity in LSc, SSc, and normal controls ${ }^{a}$.

\begin{tabular}{lccc}
\hline & \multicolumn{3}{c}{ Anti-MMP-1 Ab } \\
\cline { 2 - 4 } & IgG & IgM & IgG or IgM \\
\hline LSc (n=41) & $16(39)$ & $4(10)$ & $19(46)$ \\
GM $^{\mathrm{b}}(\mathrm{n}=12)$ & $3(25)$ & $1(8)$ & $3(25)$ \\
Linear Scl. (n=19) & $7(37)$ & $3(16)$ & $9(47)$ \\
Morphea (n=11) & $6(60)$ & $0(0)$ & $6(60)$ \\
SSc (n=37) & $7(19)$ & $9(24)$ & $18(49)$ \\
Normal (n=34) & $0(0)$ & $0(0)$ & $0(0)$ \\
\hline
\end{tabular}

${ }^{\bar{a}}$ Values are the number (\%) of patients with anti-MMP-1 Ab. IgG and IgM anti-MMP-1 Abs were determined by ELISA using anti-human IgG and IgM Abs as secondary Abs, respectively. ${ }^{\mathrm{b}} \mathrm{GM}$ = generalized morphea; Linear Scl. = linear scleroderma. 
Table 2. Clinical and serological features of patients with LSc positive for IgG anti-MMP-1 $\mathbf{A b}^{\mathrm{a}}$.

$\begin{array}{cc}\text { IgG anti-MMP-1 Ab }+ & \text { IgG anti-MMP-1 Ab - } \\ (\mathrm{n}=18) & (\mathrm{n}=23)\end{array}$

Serological

Anti-nuclear Ab (\%)

$10(56 \%)$

$16(70 \%)$

IgG antihistone $\mathrm{Ab}(\mathrm{OD})^{\mathrm{b}}$

$0.754 \pm 0.254$

$0.774 \pm 0.246$

IgG anti-ssDNA Ab (OD)

$0.683 \pm 0.29 *$

$0.485 \pm 0.170$

IgG anti-nucleosome Ab (OD)

$$
30.2 \pm 7.1 \text { * }
$$

$26.5 \pm 5.5$

Rheumatoid factor (\%)

7 (39\%)

7 (30\%)

Clinical

Sex (male/female)

$4 / 14$

$7 / 16$

Age at onset (years)

$26.3 \pm 14.6$

$17.1 \pm 18.1$

Disease duration (years)

$2.6 \pm 4.4$ *

$5.1 \pm 6.3$

No. of linear lesions

$0.67 \pm 0.69$

$1.22 \pm 1.13$

No. of plaque lesions

$2.28 \pm 2.85$

$2.43 \pm 2.86$

Total no. of lesions

$2.94 \pm 2.86$

$3.65 \pm 3.42$

No. of involved body areas

$1.89 \pm 1.37$

$2.3 \pm 1.92$

Muscle involvement (\%)

$4(22 \%)$

$5(22 \%)$

${ }^{\mathrm{a}}$ Values are mean \pm SD, unless otherwise indicated. IgG anti-MMP-1 Ab was determined by ELISA using anti-human IgG Abs as secondary Abs.

${ }^{\mathrm{b}}$ Levels of antihistone $\mathrm{Ab}$, anti-ssDNA $\mathrm{Ab}$, and anti-nucleosome $\mathrm{Ab}$ as the relative optical density (OD) were determined by specific ELISA. 
${ }^{*} \mathrm{p}<0.05$ versus patients without IgG anti-MMP-1 Ab. 


\section{FIGURE LEGENDS}

Figure 1. IgG and IgM anti-MMP-1 Ab levels in serum samples from patients with the 3 subgroups of LSc. IgG or IgM anti-MMP-1 Ab levels were determined by ELISA using human recombinant MMP-1 in patients with generalized morphea (GM), linear scleroderma (LS), or morphea (M), those with SSc, and normal controls (CTL). The short bar indicates the mean value in each group. A broken line indicates the cut-off value (mean $+2 \mathrm{SD}$ of the control samples).

Figure 2. Immunoblotting analysis of IgG anti-MMP-1 Ab in serum samples from LSc patients. Representative immunoblotting of human recombinant MMP-1 with sera from patients positive for IgG anti-MMP-1Ab by ELISA, with those negative for it, or normal controls is shown. The remaining patients or healthy individuals exhibited similar results. Lane 1, colloidal gold-stained MMP-1; lanes 2-4, serum samples from patients with LSc positive for IgG anti-MMP-1 Ab by ELISA; lane 5, a serum sample from a LSc patient negative for IgG anti-MMP-1 Ab by ELISA; and lane 6, a normal human serum. Markers for molecular weights $(\mathrm{kDa})$ are shown to the left.

Figure 3. Inhibition of MMP-1 collagenase activity by IgG isolated from serum samples that contained anti-MMP-1 Ab. IgG was purified from serum samples of LSc patients positive for IgG anti-MMP-1 Ab by ELISA and normal control. Purified IgG was incubated with MMP-1 activated by p-aminophenylmercuricacetate and MMP-1 collagenase activity (activated MMP-1) was determined using biotinylated bovine native collagen as substrate. The amount of cleaved biotinylated fragments of collagen by MMP-1 was measured by ELISA using a biotin-binding microtiter plate. MMP-1 activity is shown as a percentage of p-aminophenylmercuric acetate-activated MMP-1 that was defined as $100 \%$. Six LSc patients positive for IgG 
anti-MMP-1 Ab by ELISA and 6 healthy individuals were assessed. The data are shown as means + SD of 6 persons, each of which is the mean of the triplicate determinations.

\section{REFERENCES}

1 Jablonska S, Rodnan GP. Localized forms of scleroderma. Clin. Rheum. Dis. 1979; 5: $215-41$.

2 Sato S, Fujimoto M, Ihn $\mathrm{H}$ et al. Clinical characteristics associated with antihistone antibodies in patients with localized scleroderma. J. Am. Acad. Dermatol. 1994; 31: $567-71$.

3 Falanga V, Medsger TA, Jr., Reichlin M. Antinuclear and anti-single-stranded DNA antibodies in morphea and generalized morphea. Arch. Dermatol. 1987; 123: 350-3.

4 Sato S, Ihn H, Soma Y et al. Antihistone antibodies in patients with localized scleroderma. Arthritis Rheum. 1993; 36: 1137-41.

5 Ruffatti A, Peserico A, Glorioso S et al. Anticentromere antibody in localized scleroderma. J. Am. Acad. Dermatol. 1986; 15: 637-42.

6 Falanga V, Medsger TA, Jr., Reichlin M et al. Linear scleroderma. Clinical spectrum, prognosis, and laboratory abnormalities. Ann. Intern. Med. 1986; 104: 849-57.

7 Sato S, Kodera M, Hasegawa M et al. Antinucleosome antibody is a major autoantibody in localized scleroderma. Br. J. Dermatol. 2004; 151: 1182-8.

8 Hayakawa I, Hasegawa $\mathrm{M}$, Takehara $\mathrm{K}$ et al. Anti-DNA topoisomerase II $\alpha$ autoantibodies in localized scleroderma. Arthritis Rheum. 2004; 50: 227-32. 
9 Nagai M, Hasegawa M, Takehara $\mathrm{K}$ et al. Novel autoantibody to $\mathrm{Cu} / \mathrm{Zn}$ superoxide dismutase in patients with localized scleroderma. J. Invest. Dermatol. 2004; 122: 594-601.

10 Sato S, Fujimoto M, Hasegawa $\mathrm{M}$ et al. Anti-phospholipid antibody in localized scleroderma. Ann. Rheum. Dis. 2003; 62: 771-4.

11 Jimenez SA, Hitraya E, Varga J. Pathogenesis of scleroderma: collagen. Rheum. Dis. Clin. Nor. Am 1996; 22: 647-74.

12 Vuorio T, Makela JK, Kahari VM et al. Coordinated regulation of type I and type III collagen production and mRNA levels of pro alpha 1(I) and pro alpha 2(I) collagen in cultured morphea fibroblasts. Arch Dermatol Res 1987; 279: 154-60.

13 Kahari VM, Sandberg M, Kalimo $\mathrm{H}$ et al. Identification of fibroblasts responsible for increased collagen production in localized scleroderma by in situ hybridization. J. Invest. Dermatol. 1988; 90: 664-70.

14 Kahari VM, Saarialho-Kere U. Matrix metalloproteinases in skin. Exp. Dermatol. 1997; 6: 199-213.

15 Nagase H, Woessner JF, Jr. Matrix metalloproteinases. J. Biol. Chem. 1999; 274 : 21491-4.

16 Sato S, Hayakawa I, Hasegawa M et al. Function blocking autoantibodies against matrix metalloproteinase-1 in patients with systemic sclerosis. J. Invest. Dermatol. 2003; 120: 542-7. 
candTherapeuticCriteriaCommittee. Preliminary criteria for the classification of systemic sclerosis (scleroderma). Arthritis Rheum. 1980; 23: 581-90.

18 Krieg T, Braun-Falco O, Perlish JS et al. Collagen synthesis in generalized morphea. Arch Dermatol Res 1983; 275: 393-6.

19 Takeda K, Hatamochi A, Ueki H et al. Decreased collagenase expression in cultured systemic sclerosis fibroblasts. J. Invest. Dermatol. 1994; 103: 359-63.

20 Asano $\mathrm{Y}$, Ihn $\mathrm{H}$, Jinnin $\mathrm{M}$ et al. Involvement of alphavbeta5 integrin in the establishment of autocrine TGF-beta signaling in dermal fibroblasts derived from localized scleroderma. J Invest Dermatol 2006; 126: 1761-9.

21 El-Mofty M, Mostafa W, Esmat S et al. Suggested mechanisms of action of UVA phototherapy in morphea: a molecular study. Photodermatol Photoimmunol Photomed 2004; 20: 93-100.

22 Okano Y. Antinuclear antibody in systemic sclerosis (scleroderma). Rheum. Dis. Clin. North. Am. 1996; 22: 709-35.

23 Komocsi A, Tovari E, Kovacs $\mathrm{J}$ et al. Physical injury as a provoking factor in three patients with scleroderma. Clin. Exp. Rheumatol. 2000; 18: 622-4.

24 Trattner A, Figer A, David $M$ et al. Circumscribed scleroderma induced by postlumpectomy radiation therapy. Cancer 1991; 68: 2131-3.

25 Aiba-Kojima E, Tsuno NH, Inoue K et al. Characterization of wound drainage fluids as a source of soluble factors associated with wound healing: comparison with platelet-rich plasma and potential use in cell culture. Wound Repair Regen 2007; 15: 511-20. 

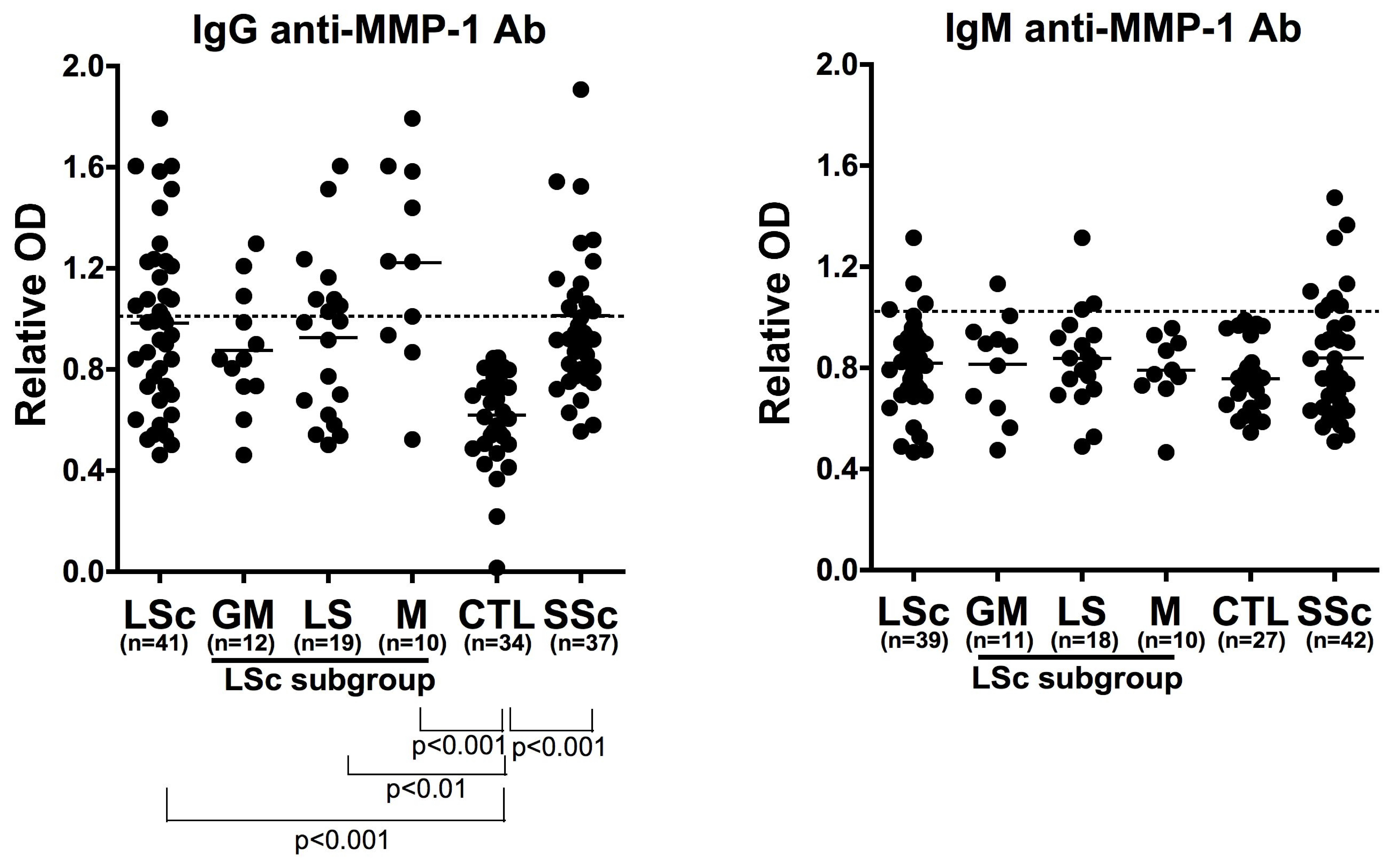

Figure 1. 


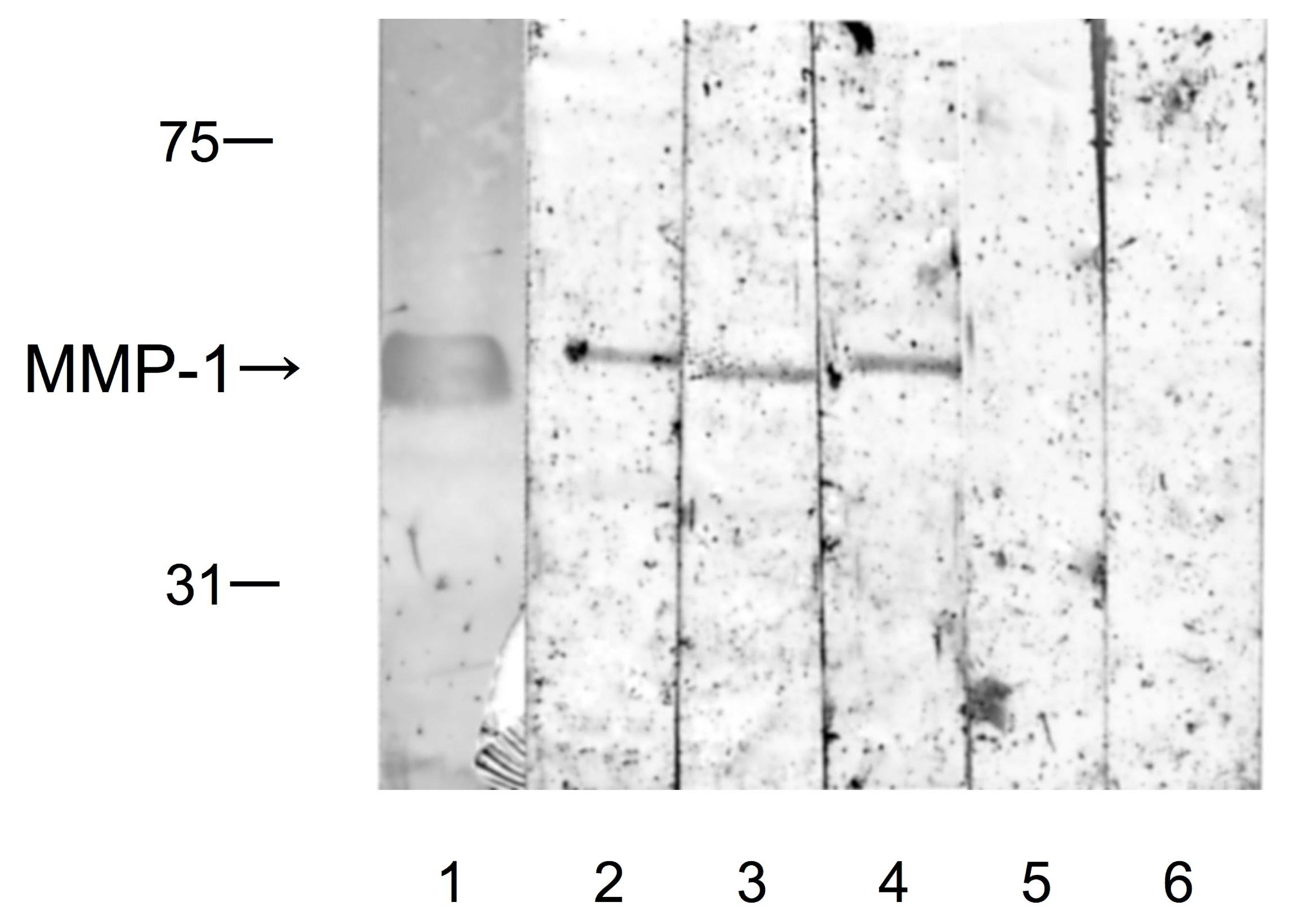

Figure.2 


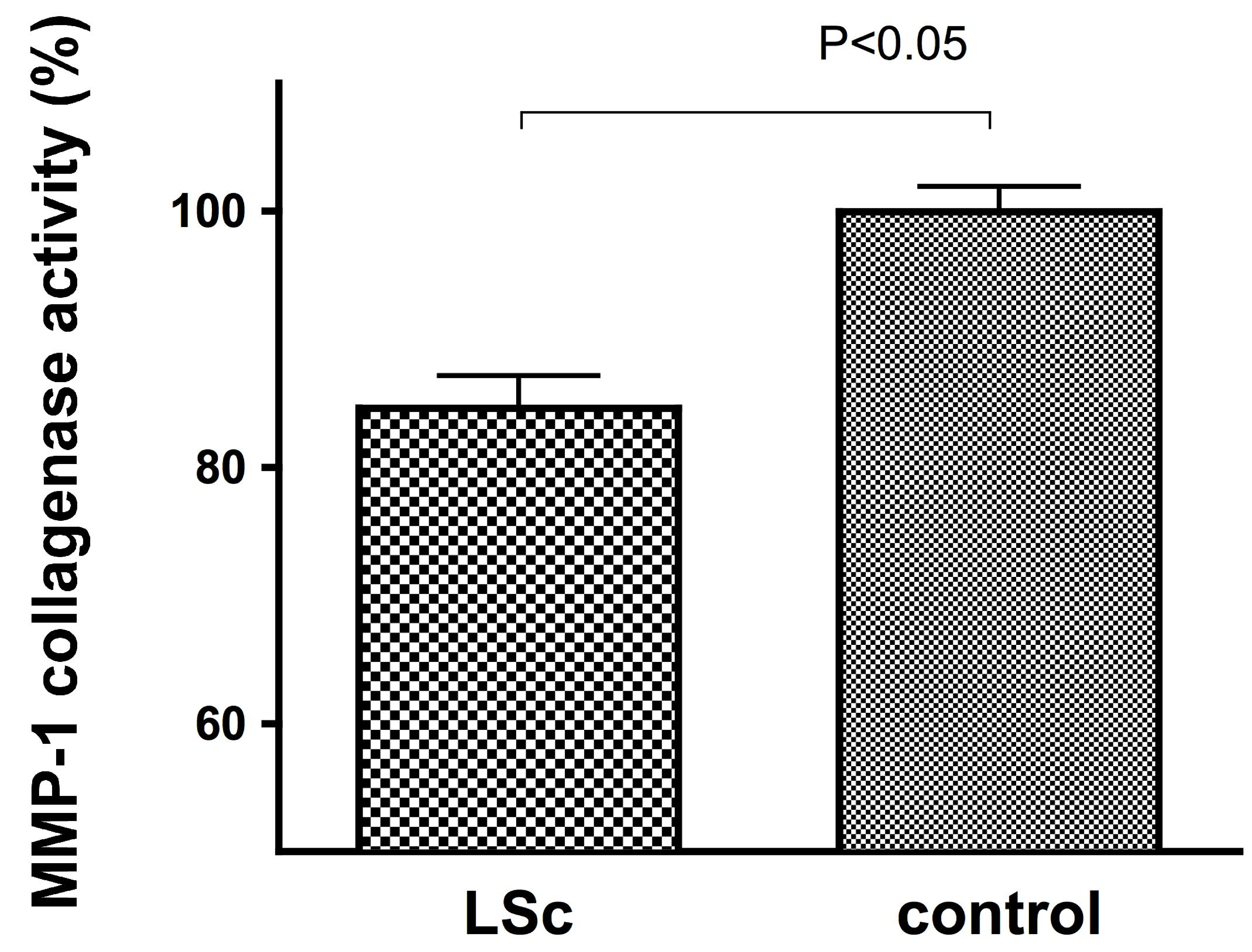

Figure.3 